\title{
Communication et formation : programmes et réception
}

Isabelle Sacré

\section{(2) OpenEdition}

1 Journals

Édition électronique

URL : http://journals.openedition.org/communicationorganisation/2085

DOI : 10.4000/communicationorganisation.2085

ISSN : $1775-3546$

Éditeur

Presses universitaires de Bordeaux

Édition imprimée

Date de publication : 1 novembre 1998

ISSN : 1168-5549

Référence électronique

Isabelle Sacré, "Communication et formation : programmes et réception », Communication et organisation [En ligne], 14 | 1998, mis en ligne le 26 mars 2012, consulté le 01 mai 2019. URL : http:// journals.openedition.org/communicationorganisation/2085; DOI : 10.4000/ communicationorganisation.2085

Ce document a été généré automatiquement le 1 mai 2019.

(c) Presses universitaires de Bordeaux 


\title{
Communication et formation : programmes et réception
}

\author{
Isabelle Sacré
}

Qu'entend-on par communication? Que désigne le vocable information-communication et quels sont les usages de ces termes ? À la base de ces questions, un constat: tous s'accordent à reconnaître la polysémie des deux termes. La communication ne désigne pas seulement cette expérience humaine immédiate et subjective, qui génère un savoir intuitif; elle recouvre aussi un ensemble de techniques, qui permet la circulation des informations, et un champ de pratiques professionnelles. Des niveaux de définition et de compréhension différents s'entrecroisent. Tout à la fois action et résultat, relation intersubjective et moyens techniques, «communication » est un mot pavillon. Il existe différentes manières d'explorer les usages pluriels de ce terme. Le sujet du présent dossier délimite pour sa part un terrain d'investigation vaste et néanmoins clairement circonscrit : celui de la formation. L'interrogation centrale devient alors celle-ci : quels sont les enseignements les plus récurrents dans les formations en communication? Quels sont les points d'ancrage du marché des savoirs transmissibles dans ce domaine?

2 Pour répondre à ces questions, je présenterai dans un premier temps une synthèse des thématiques et orientations majeures observées dans les programmes de deux formations universitaires de haut niveau: les DESS et les DEA. L'analyse parallèle de ces deux diplômes est intéressante parce que complémentaire: le premier est une filière professionnalisée, préparant directement ses diplômés à l'entrée dans la vie active; le second destine traditionnellement ses étudiants aux carrières de la recherche et de l'enseignement. Le corpus d'étude est composé de toutes les formations dont l'intitulé fait usage des termes «information» et/ou «communication». Cet argument méthodologique transgresse les découpages académiques établis qui séparent les formations selon leur discipline. Le recensement et l'analyse des programmes qui en découle ne sont pas ceux des formations affiliées à la $71^{\mathrm{e}}$ section du Conseil National des Universités - section des Sciences de l'Information et de la Communication - mise en place en $1985^{1}$. En effet, la présence du (ou des) terme(s) «information» et « communication » dans le libellé du diplôme n'est pas une condition indispensable à son 
affiliation à la $71^{\mathrm{e}}$ section. À l'inverse, c'est ce qui justifie sa prise en compte dans cette étude. Au total, quarante-deux programmes de DEA et cinquante-neuf de DESS ont été répertoriés. Cet article ne reprend évidemment pas tous les résultats de cette analyse de contenu systématique ${ }^{2}$ mais seulement les tendances significatives qui se dégagent des enseignements.

Une formation existe au travers de ses étudiants. Un diplôme sans candidat disparait. Il doit donc se faire connaître auprès des publics internes et externes à son établissement d'accueil. L'offre universitaire en troisième cycle est relativement concentrée autour de quelques pôles géographiques ${ }^{3}$ et la sélectivité des deux filières incite les candidats à déposer des demandes d'admission dans différents sites pour multiplier leurs chances. Les responsables des diplômes mènent donc une politique de communication externe afin de faire connaitre leurs formations. Le programme devient alors un outil de communication-produit, devant s'adapter au dénominateur culturel et linguistique commun à sa cible. Dans cette perspective, la seconde partie de cet article s'intéressera à la sphère des récepteurs : qui sont les destinataires des programmes de formation et quelles sont leurs attentes? Enfin, elle abordera la question des débouchés professionnels, autre message important dans la politique de communication des responsables de formation.

\section{Tendances majeures des programmes de DEA et DESS}

\section{Les sciences économiques et de gestion}

4 Les sciences économiques et de gestion sont largement sollicitées à deux niveaux étroitement imbriqués: celui des concepts et raisonnements théoriques, celui des applications à la science des organisations.

5 Les programmes de DEA s'intéressent peu à la vie quotidienne et pratique de l'entreprise comme unité de production économique. Dans cette filière, l'économie sert à révéler les enjeux sociaux, à percer les stratégies des acteurs. C'est une variable explicative des évolutions de la cité. Si l'on prend l'exemple du développement technologique, il est clair que la réflexion ne peut s'affranchir d'une analyse de ses enjeux économiques et financiers. À l'heure actuelle, la communication - définie comme un échange de sens n'implique plus seulement les véhicules historiques que sont l'écriture et l'oralité. Le sens du mot «communication» a largement étendu son territoire au secteur industriel et marchand, parce qu'il englobe toutes les activités permettant aux hommes d'échanger les informations. Le programme de DEA délaisse les activités de gestion ou de comptabilité qui mesurent au jour le jour la santé matérielle d'une entreprise. La vocation largement théorique du diplôme fait que les enseignements empruntent les concepts et principes méthodologiques aux sciences économiques à des fins heuristiques, pour étayer une démonstration et servir une analyse transversale et interdisciplinaire.

L'importance accordée aux sciences économiques et de gestion dans les programmes de DESS traduit des objectifs didactiques différents. Ce diplôme prépare l'insertion professionnelle de ses étudiants dans le tissu économique des organisations. Ceux-ci se destinent notamment à devenir des spécialistes en communication d'entreprise. Auprès des publics internes, la communication apparait comme un instrument de régulation sociale de l'entreprise et de ses composantes. La gestion des ressources humaines est appréhendée dans ce sens avec des enseignements intitulés " les tensions et les conflits " 
ou bien "pouvoir, conflit et négociation au sein des organisations", qui dévoilent sa fonction pacificatrice. Le professionnel de la communication cherche à maintenir l'homéostasie sociale dans l'entreprise. Partie prenante de la réussite économique, le personnel est l'un des principaux vecteurs de notoriété. Pour le convaincre d'exporter une image positive de son employeur, la direction de la communication interne ou des ressources humaines développe une culture d'entreprise, capable de gérer en permanence les forces contradictoires de l'ordre et du désordre au sein du collectif humain. Car si l'excès d'ordre dans une organisation conduit à l'immobilisme absolu, donc à la mort de l'entreprise, à l'inverse, l'excès de déviance des innovateurs risque de la faire éclater. C'est pourquoi la culture d'entreprise repose sur un savant dosage entre le développement d'un sentiment d'appartenance au collectif (processus fusionnel), et l'écoute des différences pour stimuler la créativité (processus de différenciation). Quant à la communication externe, elle s'adresse par définition à des publics extérieurs à l'entreprise. À travers les relations publiques, présentes dans certains programmes de DESS, elle désigne communément la communication avec l'environnement, développée autour de l'organisation comme entité. Le discours managérial tend à véhiculer l'image d'une entreprise citoyenne qui prend part à la vie sociale, assume des responsabilités collectives dépassant celles d'un acteur économique producteur de richesses. Elle parraine des actions humanitaires, soutient des opérations de solidarité nationale ou de santé publique. La filière professionnalisée qu'est le DESS initie ses étudiants aux sciences économiques et de gestion en pénétrant jusque dans le champ de leurs applications pratiques. Elle favorise une connaissance globale des entreprises - ou plus largement des organisations - et l'apprentissage de moyens d'action concrets (politique et stratégie de communication, politique commerciale, gestion managériale... etc.) pour en assurer la régulation.

\section{La veille stratégique}

7 La veille stratégique s'impose comme une problématique majeure dans les programmes de DEA et de DESS. Derrière ce vocable se cache une nouvelle version du management des systèmes d'informations des entreprises, que l'on peut définir comme «le système qui fournit des informations supportant les opérations, la gestion et les prises de décision dans une organisation sociale $»^{4}$. La veille stratégique permet l'accès à une information spécialisée, qui favorise l'innovation dans les procédés de fabrication et de gestion, dans l'organisation du travail et le management des entreprises. Même si l'expression est relativement nouvelle dans le vocabulaire entrepreneurial, elle est déjà polysémique et recouvre en fait « diverses actions visant à maintenir la connaissance la plus exhaustive possible de l'évolution que subit l'environnement technico-économique d'une entreprise, afin de pouvoir anticiper les décisions les plus adéquates au maintien de sa performance " 5. Plus qu'un outil d'organisation, la veille stratégique est par conséquent un instrument de prévision, une extrapolation de phénomènes scientifiques à partir de données passées ou actuelles. En intégrant la notion d'intelligence stratégique, elle évoque la bataille économique que se livrent les entreprises par systèmes d'informations interposés. Cette surveillance de l'environnement technico-économique peut se limiter dans le cas d'une entreprise au seul niveau concurrentiel ayant l'impact le plus immédiat et le plus direct sur l'activité commerciale. La veille stratégique est alors un outil de contrôle et de gestion des connaissances, au service du management. Mais les acteurs étatiques sont a prioritout autant concernés par la menace de l'espionnage informationnel et de l'agressivité 
commerciale. La veille stratégique devient dans ce cas un projet de surveillance systématique d'un environnement élargi, dans ses rouages économiques, politiques et sociaux. Les différentes fonctions de la veille stratégique mentionnées dans les programmes peuvent sembler contradictoires mais elles sont en réalité étroitement imbriquées: favoriser et optimiser l'accès à l'information d'une part (fonctions documentaire, de formation et de médiation), contrôler et verrouiller ce même accès d'autre part (fonctions de sécurité, de surveillance et de protection). Cette ambivalence est une constante dans les usages sociaux des techniques de communication. Reprenons l'exemple classique du répondeur téléphonique: d'un côté il assure une connexion permanente avec l'extérieur, de l'autre c'est un moyen de filtrer ses appels. Cette contradiction apparente n'est pas sans rappeler l'expérience subjective de la communication directe: attirance et répulsion composent ensemble et fondamentalement la relation à autrui.

\section{Les sciences juridiques}

8 Le droit des médias et des technologies informatisées est sans conteste l'enseignement juridique en DEA le plus évoqué.

9 Les nouvelles technologies interrogent les sciences juridiques. Mais si les autoroutes de l'information soulèvent depuis quelques années débats et polémiques sur la nécessité - et les difficultés - d'une juridiction spécifique et uniforme, l'ingérence du droit dans le champ de l'information et de la communication n'est pas si récente. Les médias traditionnels sont des objets de recherche privilégiés pour des approches sectorielles droit de l'audiovisuel, droit de la presse écrite - qui reflètent la rigueur et le cloisonnement caractéristiques des sciences juridiques. Certains programmes se penchent sur l'histoire du droit et sur ses dimensions européenne et internationale. Un seul d'entre eux évoque la question des droits d'auteurs, explicite comme on le verra dans plusieurs programmes de DESS. La rencontre entre le droit et la communication ne se déroule pas toujours sans heurts. Pour Serge Regourd, «le droit est autant malmené par la communication que celle-ci se fonde sur des concepts et traits d'identification qui sont radicalement antinomiques de l'épistémologie juridique $»^{6}$. Alors que le droit distingue scrupuleusement le droit de l'audiovisuel de celui du cinéma, ou encore le droit de la publicité de celui de l'affichage, la communication abonde dans la confusion des genres et fait l'amalgame entre métiers de la presse écrite et audiovisuelle, relations publiques et communication interne. Les évolutions technologiques déstabilisent également les cadres législatifs traditionnels, qui se révèlent inadaptés aux nouveaux usages en matière de communication. Dans l'approche juridique, le cloisonnement de départ est tel qu'à un procédé technique doivent correspondre un usage et un droit uniques. Tout se passerait bien si les pratiques ne remettaient pas rapidement en question cette idée de monofonctionnalité. Prenons le cas du téléphone. C'est aujourd'hui un outil d'usage à la fois privé et professionnel et son exploitation à des fins commerciales pose inévitablement la question du respect de la vie privée. Même si nos sociétés n'ont pas attendu l'avènement de l'informatique pour ficher et contrôler les individus ${ }^{7}$, les performances de l'ordinateur augmentent considérablement les capacités de traitement des informations et les possibilités d'interconnexion de fichiers. Les sociétés de marketing téléphonique ont d'ailleurs su tirer profit de l'absence de réglementation spécifique. En fait, la difficulté majeure vient de ce que l'émergence de nouveaux usages précède souvent la 
jurisprudence ; c'est pourquoi le législateur est contraint d'intervenir en permanence dans une course-poursuite à l'évolution des techniques et à la créativité sans cesse renouvelée des usagers. L'intérêt manifesté pour la propriété littéraire et artistique dans les programmes de DESS peut s'expliquer par le fait que ce secteur connaisse depuis quelques années une véritable mutation. En s'ouvrant au domaine de l'industrie, le droit modifie considérablement la sociologie classique des auteurs. S'ils ressemblent dans certains cas à des inventeurs, dans d'autres, ce sont plutôt des prestataires de services comme des concepteurs de logiciels ou des compilateurs d'annuaires. Cette révolution dans le concept d'auteur suscite des interrogations essentielles, notamment sur la protection de ces productions de l'esprit : rémunère-t-on véritablement l'auteur lorsque l'on protège le monopole d'une équipe d'informaticiens professionnels sur une banque de données qu'elle a créée ? N'est-ce pas plutôt au bénéfice de l'investisseur économique du projet? Même si elle soulève plus de questions et de polémiques qu'elle n'apporte de réponses à l'heure actuelle, l'ingérence du droit dans les programmes de troisièmes cycles universitaires est réelle. Les sciences juridiques font désormais partie des disciplines incontournables pour étudier les champs de l'information et de la communication. On remarquera au passage que seul un enseignement en DEA réunit les termes « information » et « communication » dans une seule proposition. Ce clivage témoigne-til de la précision et de la rigueur caractéristiques des classifications juridiques?

\section{Enseignements méthodologiques et techniques : le paradigme du calcul}

10 La tendance générale est au calcul et à la quantification. Les méthodes enseignées doivent gérer la masse de données plus que le sens des informations. Pour cela, l'homme sollicite l'aide d'un cerveau électronique, capable de traiter un maximum d'énergies en un minimum de temps. Dans ces programmes de troisièmes cycles - DEA et DESS confondus l'effort se concentre donc sur la manipulation du support informatique pour un traitement quantitatif des données. Cette surabondance informationnelle semble aller de soi: les méthodes de recherche et d'accès sont plus faiblement évoquées dans les enseignements. La disponibilité théorique de l'information devient de fait un accès réel, effectif. Les méthodes d'enquêtes qualitatives issues des sciences sociales sont également quelque peu oubliées dans le contenu manifeste des programmes. Ceci étant, on sait que les deux méthodes (quantitatives et qualitatives) sont complémentaires plus qu'elles ne s'opposent dans une recherche. Dans le registre qualitatif, l'entretien est certes plus à même de restituer des objets d'étude difficilement mesurables comme par exemple les composantes affectives. Mais on sait aussi que de nombreuses enquêtes quantitatives reposent en partie sur des catégories plus ou moins arbitraires et qualitatives.

11 Les techniques informatisées sont prédominantes dans les enseignements des deux diplômes. Leur comparaison montre que les programmes de DESS restent à un plus grand degré de généralité que ceux de DEA, dans lesquels le vocabulaire est souvent plus spécifique et pointu. Dans tous les cas, plus de la moitié des enseignements techniques est centrée sur ces supports élaborés pour recevoir et gérer une grande quantité d'informations. Des cours élémentaires sont même parfois prévus dans certaines formations pour homogénéiser les connaissances et familiariser le plus grand nombre au maniement des nouveaux outils d'information et de communication's. Si l'ordinateur permet le traitement massif des informations, il ajoute aussi la vitesse dans la 
compilation, la consultation et le traitement des données pour des opérations complexes. L'écriture a introduit la linéarité, les distinctions fortes de cause à effet, les notions d'avant et d'après. L'informatique repose quant à elle sur l'immédiateté et la réversibilité : l'outil prend le pas sur l'objet. Les entreprises entrent dans une logique de l'immatériel en investissant de plus en plus dans les nouvelles technologies. Elles s'intéressent aux capacités d'innovation et plus seulement de production. C'est la recherche d'une rentabilité globale, plus que d'une productivité au travail dans le sens strict du terme. Mais ces investissements techniques sont largement tributaires des investissements en connaissances, capables de concevoir et d'exploiter les ressources technologiques. Car c'est bien le savoir de l'homme qui est à la fois la matière première et le produit fini dans cette course à la performance. Ainsi, au-delà de leur interdisciplinarité, les programmes semblent fortement imprégnés du «paradigme du calcul»'. Argument ontologique ou simple métaphore pour décrire l'univers scientifique contemporain ? Cette question pourrait être le point de départ d'une autre réflexion...

$\mathrm{Au}$ terme de cette analyse des formations, on constate une certaine forme de marginalisation des phénomènes humains et sociaux associés à la communication. Les programmes écartent le plus souvent l'univers des relations interpersonnelles, nourri des apports de la psychologie, de la linguistique ou de l'anthropologie. L'absence d'outils de développement de la personnalité est regrettable, car on sait que les qualités individuelles sont capitales dans le recrutement des professionnels de la communication à ce niveau de diplôme. Les responsabilités qui leur sont confiées en termes d'organisation et de conception sont telles que la personnalité, les compétences relationnelles, l'éthique sont autant d'atouts. La majeure partie des programmes appréhende le champ de l'information-communication à travers ses seuls outils. La communication devient organisée pour toucher le plus grand nombre dans un environnement élargi. Les moyens déployés à cette fin se technicisent pour une meilleure performance et une rentabilité accrue. Communiquer est devenu un impératif fonctionnel dans un contexte de mondialisation des échanges. Après la société de consommation vient celle de la communication ou plus justement de son instrumentalisation. L'information devient potentiellement accessible à tous dans le cyberespace. La confusion s'installe entre interactivité et communication, échange d'informations et partage de significations. L'échange direct - aussi complexe soit-il - est désormais possible sans intermédiaire, si ce n'est un clavier d'ordinateur. Tout se passe comme si les inforoutes abolissaient les hiérarchies de compétences et les distances entre les savoirs. L'information devient simultanément connaissance. Et cette équation crée l'illusion d'une même capacité de décryptage et d'interprétation chez chacun d'entre nous, d'un désir de savoir identique.

\section{Programme de formation et communication-produit}

De manière générale, une organisation définit une stratégie de communication afin de se faire connaître auprès de ses publics et pour véhiculer une image positive d'elle-même capable de susciter leur adhésion. Un des moyens possibles consiste à développer une communication-produit. Dans cette perspective, les responsables de formation élaborent des programmes présentant leurs diplômes habilités par le ministère et leurs spécificités. Les destinataires de ce message sont prioritairement les étudiants, ou plus précisément dans le cas présent les candidats potentiels à la préparation d'un DEA ou d'un DESS. 


\section{À l'intersection de deux logiques} ministère de l'éducation nationale ${ }^{10}$ : chaque formation doit satisfaire certaines conditions pour recevoir une habilitation au titre de diplôme universitaire français. « La part essentielle d'une formation de DEA doit être consacrée à l'initiation à la recherche ; l'étudiant bénéficie d'enseignements théoriques et méthodologiques, et d'une initiation aux techniques de recherche dont le volume annuel n'excédera pas 200 heures ${ }^{11}$. Le DESS doit pour sa part inclure des «enseignements théoriques, des enseignements pratiques et un stage $»^{12}$. Les experts du ministère sont donc les premiers récepteurs du message contenu dans le programme de formation. Après avis du CNESER ${ }^{13}$, le dossier de DEA est étudié par le ou les Groupes d'Études Techniques compétents ; celui de DESS est examiné par un Comité d'Expertise Pédagogique des Projets d'Établissements.

16 La seconde exigence est une lisibilité optimale des contenus et objectifs de formation pour un public averti ou non. Élaboré et légitimé par des spécialistes, le programme se veut également accessible hors du cercle des initiés. Même si une formation de troisième cycle mobilise très largement les acquis antérieurs, elle propose à ses candidats un nouvel ensemble d'apprentissages d'ordre cognitif, comportemental et attitudinal. En outre, si ces formations s'adressent par définition aux diplômés de niveau bac +4 ou équivalent possédant déjà une certaine culture universitaire, les candidatures aux DEA et DESS ici recensés n'émanent pas obligatoirement de personnes déjà formées à la communication. Prenons deux exemples. Pour solliciter leur intégration dans le DEA Sciences de l'Information et de la Communication à Paris 3, les candidats doivent joindre à leur dossier « un diplôme de second cycle attestant de l'obtention de la maîtrise ». Dans ce premier cas, les conditions d'admission laissent supposer que le niveau d'études atteint importe beaucoup plus que la discipline d'origine. Quant au DESS Information et Communication préparé à Lyon 3, il a pour vocation de « permettre à des étudiants ayant une formation supérieure dans un autre domaine (Lettres, Droit, Gestion, Sciences,...) d'acquérir une seconde compétence ». Ces deux exemples ne sont pas des cas isolés ${ }^{14}$. De nombreux diplômes de troisième cycle en communication misent sur la transversalité et l'interdisciplinarité de leur champ d'investigation et recrutent à la fois des candidats déjà initiés et des diplômés en économie, gestion, lettres ou mathématiques.

\section{Une cible étudiante segmentée}

17 La cible étudiante pour un programme de DEA ou de DESS peut donc être divisée en deux sous-ensembles au moins.

18 - Une population possédant déjà une formation de base de niveau bac+4 en informationcommunication et qui recherche un diplôme de spécialisation comme le DESS ou un troisième cycle d'approfondissement comme le DEA. Un diplômé en Information et Communication sociale va ainsi postuler pour un DESS Communication des organisations ou un DEA en Sciences de l'information et de la Communication. Ce cas de figure est celui qui préside dans la plupart des autres disciplines académiques : un diplômé bac +4 en Psychologie se spécialise en choisissant un troisième cycle professionnalisé de 
Psychologie du travail ; une Maîtrise de Droit est la première condition d'admissibilité dans un DESS de Droit international. s'assurer de la meilleure réception possible de son message en recherchant le plus important dénominateur linguistique commun à la population visée. Pour le premier segment - population initiée au champ de l'information-communication - le programme de la formation fera usage d'un vocabulaire technique et scientifique spécialisé, qui évincera les candidatures des dilettantes en raison d'une trop grande opacité. C'est tout particulièrement le cas des troisièmes cycles consacrés à la conception, au traitement et à la diffusion de l'information, qui réclament des connaissances très techniques ${ }^{15}$. Pour le second segment - public novice en information-communication - la stratégie de communication sera différente. Le programme de formation emploiera souvent un vocabulaire très général pour une accessibilité élargie. Afin d'encourager des candidatures de différents horizons disciplinaires, le contenu sera flexible pour une lisibilité maximale, même si c'est au prix d'un contenu informationnel assez limité. La recherche du dénominateur commun expliquerait donc en partie au moins le caractère générique de certains enseignements annoncés dans les programmes. Les modules méthodologiques en DEA sont à ce titre tout particulièrement concernés : sur quarantedeux programmes analysés, on a relevé dix-huit occurrences de l'intitulé «méthodologie (s)» et quinze «initiation à la recherche», sans autre précision sur le contenu de ces cours.

\section{Le programme côté réception}

21 On peut alors se poser la question de savoir si ce faible contenu informationnel dans certains programmes est ressenti comme tel par les étudiants auxquels ils sont destinés. Mon expérience de jury dans la sélection des candidats au DESS Communication et Jeunesse de Bordeaux 3 m'encourage à penser que leur principale préoccupation est ailleurs. En effet, une part non négligeable des postulants semble plus intéressée par le niveau d'études accessible avec un troisième cycle que par le contenu distinctif de tel ou tel diplôme. La motivation dominante n'est pas toujours la réalisation d'un projet professionnel réfléchi, mais plutôt la recherche d'une assurance supplémentaire contre les risques du chômage. En effet, malgré une dégradation globale depuis dix ans, on sait que les taux de chômage baissent à mesure que le niveau d'études atteint augmente ${ }^{16}$. En outre, beaucoup d'étudiants attendent d'un DESS qu'il leur apporte le projet professionnel qui leur fait défaut, alors que cette formation professionnalisée est justement là pour satisfaire un besoin déjà ressenti. Selon François Lebas, responsable du SUIO ${ }^{17}$ de l'Université de Bordeaux 3, trop d'étudiants concentrent en fait leur intérêt sur des informations pratiques comme les dates ou la nature des sélections, sans se pencher précisément sur le programme des enseignements. Il y a certes les boulimiques de l'information mais ils semblent «incapables de définir des critères de choix ou des critères d'analyse de cette formation par rapport à leur projet $»^{18}$. C'est pourquoi le 
travail des interfaces que sont les SUIO est avant tout d'aider chacun d'eux à définir ce qu'il cherche. Assister l'étudiant dans la définition d'un projet personnel d'orientation mobilise très largement le temps et l'énergie de ces professionnels. Et ce rôle vient en amont de celui de relais dans la circulation des informations sur les formations. Plus que le programme des cours, ce sont les perspectives d'emploi après la formation qui intéressent les personnes à la recherche d'un troisième cycle universitaire, même si l'on sait que de plus en plus - hausse générale du niveau d'études et pénurie de l'emploi obligent - le facteur discriminant face au travail est l'individu lui-même plus que sa formation. Ce dernier point est d'autant plus vérifiable que les métiers de l'informationcommunication sont des professions non statutaires. Contrairement à un médecin ou à un avocat, un journaliste n'est pas obligatoirement diplômé en journalisme et un chargé de communication interne n'est pas toujours passé par une école de communication. Peutêtre les choses évolueront-elles à mesure que les métiers s'organiseront, mais à l'heure actuelle, il n'y a pas de relation adéquationniste entre formation et emploi. Le DESS attire les candidatures parce qu'il est un diplôme professionnalisé préparant directement l'entrée sur le marché du travail. Mais il ne prétend pas garantir l'employabilité de tous ses diplômés, a fortiorisi ceux-ci n'ont pas personnellement mûri un projet professionnel.

\section{La communication sur les débouchés professionnels}

Le contenu des enseignements n'est pas le seul message communiqué par les responsables de formation. Les débouchés professionnels constituent un autre sujet important développé dans les plaquettes de présentation des diplômes. Les DESS y accordent une attention toute particulière mais - et c'est un fait plus inhabituel - certaines plaquettes de DEA évoquent également des perspectives d'emploi en dehors des traditionnelles voies de l'enseignement et de la recherche. C'est le cas par exemple des diplômes centrés sur l'information spécialisée, très présents dans le corpus. Axée sur la recherche, la formation doctorale répond ainsi aux besoins ressentis sur le marché du travail, au niveau de la conception et de l'expertise des systèmes d'information et plus généralement en matière de veille technologique. Mais c'est aussi le cas de formations plus généralistes comme le DEA en Sciences de l'Information et de la Communication du CELSA ${ }^{19}$ dont « la politique de recherche est déterminée, conformément à ses choix pédagogiques fondamentaux, par sa double vocation universitaire et professionnelle». La question de l'insertion professionnelle des étudiants n'est pas nouvelle. Déjà présente dans la loi Savary du 26 janvier 1984 sur la professionnalisation des universités, elle est réitérée dans les textes suivants ${ }^{20}$. Elle se manifeste également dans la pression sociale de résorption du chômage et l'inquiétude croissante des étudiants face à leur avenir professionnel. L'articulation entre l'offre et la demande de qualifications est elle-même problématique lorsque l'on constate que certains emplois restent inoccupés, faute de candidats compétents. C'est pourquoi les responsables universitaires doivent en permanence travailler à l'ajustement de leurs formations aux évolutions du marché du travail, pour finalement préparer au mieux l'insertion professionnelle des diplômés.

Pour atteindre cet objectif, l'heure est à la réflexion concertée. Salons spécialisés ${ }^{21}$, colloques, universités d'été2 ${ }^{2}$, constituent des lieux d'échange, des espaces de dialogue et de confrontation entre universitaires et professionnels. Ce sont aussi des actions de communication externe porteuses en termes d'image et de notoriété. Au sein des formations, des relations de partenariat se développent. Les acteurs économiques et 
professionnels interviennent dans les projets de nouveaux diplômes ${ }^{23}$. Ils exercent des responsabilités pédagogiques dans les commissions de spécialistes notamment et en matière d'enseignement. Des conventions-cadres sont signées entre les responsables de formation et les entreprises. Le partenariat devient financier lorsque celles-ci versent tout ou partie de la taxe d'apprentissage au bénéfice du fonctionnement des formations. Les étudiants ressentent également les effets de cette ouverture de l'Université sur le monde extérieur. Les opportunités de réaliser des stages en entreprise et sous convention jalonnent les cursus d'études. Les services d'offres d'emploi ont leur place sur les panneaux d'affichage. Des associations d'anciens élèves sont créées pour stimuler les échanges entre les étudiants et les diplômés professionnellement intégrés.

Dans le domaine de l'information-communication, le dialogue et la collaboration entre universitaires et professionnels sont particulièrement importants. C'est un secteur estimé porteur d'emplois parce que le recours aux fonctions d'information et de communication s'étend de plus en plus à l'ensemble des métiers. Ces fonctions transgressent les clivages sectoriels : on communique dans tous les domaines d'activités et l'échange d'informations est omniprésent. Jusque là, la situation n'est pas nouvelle : d'autres professions comme celle de secrétaire ou de comptable sont concernées. Mais à l'inverse de ces deux exemples, communiquer n'est pas seulement l'affaire d'une personne spécialisée dans l'entreprise. Désormais, tout le monde doit avoir des compétences en informationcommunication, à commencer par la maitrise des nouvelles technologies. Au-delà des fonctions, on constate donc une extension de l'usage des outils de communication, dans l'ensemble des activités humaines : à son travail, à son domicile, à titre privé et collectif. Fonctions, outils : la confusion s'installe entre l'émergence de nouveaux métiers comme celui de chargé de communication qui justifie la mise en place de formations spécialisées, et l'apparition de nouvelles modalités d'exercice des professions qui nécessite d'introduire des enseignements de sensibilisation à l'information-communication dans toutes les filières et à tous les niveaux de formation. Cette ambiguïté impose aux responsables de formation de connaître et de communiquer les perspectives d'emploi pour les futurs diplômés. Cette communication sur les débouchés est encore plus justifiée quand il s'agit de formations dont la visée professionnelle est à spectre large, c'est-à-dire avec des possibilités d'insertion très variées ${ }^{24}$. Le marché de l'emploi en informationcommunication est un marché aux limites imprécises, encore jeune et très fortement évolutif. Face à cette dynamique, la veille stratégique s'impose de la part des responsables de formation. Le contact avec les praticiens est un moyen de rester en prise avec les réalités concrètes et quotidiennes.

À travers les programmes de DEA et de DESS, le marché des savoirs transmissibles affirme la transversalité du champ de l'information-communication. L'ingérence des sciences économiques et de gestion, des sciences logiques dans la thématique de la veille technologique, ou encore des sciences juridiques, montre l'importance de son territoire mais aussi l'imprécision de ses frontières. Plus qu'un objet d'étude spécifique, c'est une manière de voir, de penser la vie des organisations, une reformulation (théorique et méthodologique) de problèmes déjà soulevés par d'autres disciplines plus anciennes. L'absence de monopole crée une situation de concurrence que, d'une certaine manière, les candidats aux troisièmes cycles d'études universitaires, déjà initiés au domaine de l'information-communication, doivent affronter à un double niveau. En premier lieu, la concurrence s'opère sur le marché de l'offre de formation qui, au nom de la double compétence, recrute en partie des étudiants formés dans d'autres disciplines. La seconde 
forme de concurrence s'exerce sur le marché de l'emploi et elle est liée au caractère non réglementaire des métiers de l'information-communication évoqué précédemment. $\mathrm{D}$ suffit de se pencher sur les offres d'emploi spécialisées pour réaliser que les diplômés universitaires en communication ne sont pas toujours recrutés en priorité sur des postes pour lesquels paradoxalement ils ont été formés. Si l'on s'en tient au texte des annonces, les formations commerciales, en marketing ou en gestion semblent être souvent des profils plus appréciés des employeurs. Communiquer sur les débouchés professionnels est sans doute une nécessité pour répondre à la forte demande sociale. Mais ce message ne doit pas se substituer à tous les autres, sous peine d'entretenir de dangereuses illusions, comme le font certaines écoles privées. Une formation en communication - aussi réputée soit-elle - n'est jamais à elle seule un passeport pour l'emploi. Et c'est dans le contenu des programmes que l'on peut évaluer la pertinence ou non des perspectives de travail présumées.

\section{Ouvrages collectifs}

Vers la société de l'information : savoirs, pratiques, médiation, ss la dir. de R Delmas et $\mathrm{F}$. Massit-Folléa, Paris, Apogée, 1995, 204 p.

\section{BIBLIOGRAPHIE}

BOSQUET René, Fondements de la performance humaine dans l'entreprise,Paris, Les Éditions d'Organisation, 1989, $125 \mathrm{p}$.

BOUDON Raymond et Bourricaud François, Dictionnaire critique de la sociologie, Paris, PUF, 1990, $714 \mathrm{p}$.

FAVE-BONNET Marie-Françoise, "L’Université : état des lieux », in Èduqueret former, Paris, Éditions Sciences Humaines, 1997, 540 p.

FOUCAULT Michel, Surveiller et punir : naissance de la prison, Paris, Gallimard, 1993, 360 p.

GALINON-MELENEC Béatrice, Projet et communication dans les universités, Paris, Les Éditions d'Organisation, 1991, $225 \mathrm{p}$.

GAUDRAT Philippe, «Propriété des créations : l'auteur mutant », in Dictionnaire critique de la communication, Paris,PUF, 1993,1 780 p.

GRAWITZ Madeleine, Lexique des sciences sociales, Paris, Dalloz, 1994, 402 p.

HUISMANN Denis et LOUGOVOY Constantin, Traité de relations publiques, Paris, PUF, 1981, 641 p.

NICOLAS Anne-Gaëlle, « Rencontre du $3^{\mathrm{e}}$ cycle », in Transfac, nº 100, février 1996, pp. 34-36.

QUEAU Philippe, «Internet, média pour le XxI ${ }^{\mathrm{e}}$ siècle », in Le Monde Diplomatique, $\mathrm{n}^{\circ}$ 27, 1995, pp. 47-48.

REGOURD Serge, « Le droit au risque de la communication », in Cinémaction, n 63,1992, pp. 95-98. 
SACRE Isabelle, Information et/ou communication : pluralité des usages dans les univers théoriques et les formations, Thèse de doctorat, université Michel de Montaigne de Bordeaux 3,19 \%, 776 p.

SACRE Isabelle, Formations en information communication,Paris, L'Harmattan, 1998,176 p.

TARDIEU Hubert, « Systèmes d'information et de gestion », in Dictionnaire critique de la communication,Paris, PUF, 1993,1780 p.

VASSEUR Frédéric, Les médias du futur, Paris, PUF, Coll. Que-Sais-Je, 1992, 227 p.

D'une science à l'autre : des concepts nomades, ss la dir. d'Isabelle Stengers, Paris, Seuil, 1988, 388 p.

Quelques diagnostics et remèdes urgents pour une université en péril, ss la dir. du bureau de l'ARESER, Paris, Liber-raisons d'agir, 1997,124 p.

Les sciences de l'information et de la communication, Rapport du Comité National d'Évaluation, Paris, $1993,125 \mathrm{p}$.

\section{Textes de loi}

Loi Savary du 26 janvier 1984 sur la professionnalisation des universités.

Circulaire ministérielle du 24 mars 1989.

Arrêté du 30 mars 1992 relatif aux études de troisième cycle.

Décret 96-16 du 10 janvier 1996.

\section{Recensement des formations}

Annuaire national des DEA,Ministère de l'Enseignement Supérieur et de la Recherche, Paris, éditions septembre 93 et 96-97.

Liste des DESS,Ministère de l'Enseignement Supérieur et de la Recherche, Paris, éditions 93-94 et 97. Fiches $d u$ CIDJ,Paris, éditions juin 93 et juin 96

\section{NOTES}

1. En réalité, seulement $58,4 \%$ des formations recensées ici sont affiliées a la $71^{\mathrm{e}}$ section des Sciences de l'Information et de la Communication, nouvelle dénomination de la $52^{\mathrm{e}}$ section mise en place en 1974.

2. (Sacré, 1996, 1998).

3. L'Ile-de-France propose à elle seule près d'un tiers des DEA et DESS du corpus présenté plus haut.

4. (Tardieu. 1993)

5. Vers la société de l'information : savoirs, pratiques, médiation.

6. (Regourd, 1992).

7. Cf notamment l'ouvrage de Michel Foucault, Surveiller et punir.

8. Philippe Queau estime à plus de 30 millions le nombre d'utilisateurs d'Internet dans le monde et un taux de croissance exponentiel équivalent à plus de $1700 \%$ pour le principal réseau le World Wide Web (WWW) en 1994 » ( Internet, média pour le XxI ${ }^{\mathrm{e}}$ siècle» in Le Monde Diplomatique, (27), Août 1995, p. 47)

9. D'une science à l'autre : des concepts nomades. 
10. La mise en place d'un diplôme de DEA. ou de DESS est précédée d'une demande d'habilitation établie sur la base d'un dossier administratif et pédagogique, lequel est examiné au sein de l'université d'accueil avant d'être expertisé au niveau ministériel. À l'issue de cette procédure, l'habilitation est accordée ou non, pour une durée de 2 à 4 ans.

11. Art. 15 de l'arrêté du 30 mars 1992 relatif aux études de troisième cycle.

12. Art. 10 de l'arrêté du 30 mars 1992 relatif aux études de troisième cycle.

13. Conseil National de l'Enseignement Supérieur et de la Recherche

14. Tous deux relèvent de la $71^{\mathrm{e}}$ section des Sciences de l'Information et de la Communication.

15. Exemples : DEA « Informa bon Scientifique et Technique » ou DESS « Audit et conception des systèmes d'information ».

16. (Nicolas, 19\%).

17. Service Universitaire d'Information et d'Orientation.

18. Entretien avec François Lebas, 9 juillet 1998

19. Institut des hautes études en sciences de l'information et de la communication.

20. Cf. par exemple la circulaire ministérielle du 24 mars 1989 qui instaure une politique contractuelle entre l'Etat et les établissements et le décret 96-16 du 10 janvier 1996

21. Exemples : Aquitec (salon de la formation, des métiers et de l'emploi en Aquitaine) et le Salon des troisièmes cycles à Paris

22. Cf Université d'été de la communication à Carcans-Maubuisson en Gironde.

23. Un dossier de demande d'habilitation en DESS doit faire valoir le soutien des professionnels du secteur d'activité concerné.

24. Exemples : la communication d'entreprise, l'information spécialisée

\section{RÉSUMÉS}

L'auteur s'intéresse aux différents sens accordés aux termes «information » et « communication ». Dans la première partie de cet article, la question est plus précisément de savoir ce que l'on enseigne aux personnes qui choisissent de préparer un diplôme en information-communication. A partir d'un recensement des formations méthodologiquement argumenté dans l'introduction, une synthèse des disciplines et thématiques observées dans les programmes de DEA et DESS est proposée, comme une configuration possible du marché des savoirs transmissibles. Parce que le programme d'une formation est aussi un produit de communication contenant un message et destiné à une cible, la seconde partie évoque la question de sa réception par les étudiants et celle de la communication sur les débouchés professionnels.

The author takes an interest in differents senses and uses of "information" and «communication» words. Considering that information and communication are two subjects teached at french school, he describes and explains the most current knowledges in two higher education's graduates. In the first place, the article purposes a synthesis of university syllabuses. Secondly, the author compares a syllabus to a product of communication and he raises the question of reception by students, which want to study information's and communication's sciences. Lastly, he wonders about professional opportunities after education's time. 
INDEX

Mots-clés : programmes, université, diplôme, information

\section{AUTEUR}

\section{ISABELLE SACRÉ}

Isabelle Sacré, docteur en Sciences de l'Information et de la Communication, pose dans ses recherches la question des usages du vocable « information-communication ».

L'analyse des programmes de formation - qui sont également des produits de communication - lui permet de dégager certaines thématiques dominantes qui structurent le marché des savoirs dans sa discipline. L'auteur vient de publier Formations en information communication aux éditions L'Harmattan. 